\title{
No evidence for rule-based processing in the inverse base-rate effect
}

\author{
Koen LAMberts AND Christopher Kent \\ University of Warwick, Coventry, England
}

\begin{abstract}
The inverse base-rate effect in categorization (Medin \& Edelson, 1988) arises when participants assign an ambiguous stimulus to a category that occurred less frequently than an alternative category, against the principles of Bayesian decision making. In the experiment reported in this article, rule-based and attention-shifting accounts of the inverse base-rate effect were evaluated. Participants completed a categorization task, known to produce the inverse base-rate effect, under standard conditions, under time pressure, and with a secondary task load. The inverse base-rate effect persisted under severe time pressure and under secondary task load. The results provided no evidence for the role of rule-based processes in producing the inverse base-rate effect. The data from the experiment are compatible with an attention-shifting account.
\end{abstract}

The inverse base-rate effect is the finding that participants in a categorization task sometimes assign an ambiguous stimulus to a category that occurred less frequently than other categories, against the normative specification of Bayesian decision theory. The effect was first reported by Medin and Edelson (1988) in a simulated medical diagnosis task. The structure of the training stimuli used in the task is shown in Table 1. Symptoms $P C_{a}, P C_{b}$, and $P C_{c}$ were perfect predictors of a common disease, symptoms $P R_{a}, P R_{b}$, and $P R_{c}$ were perfect predictors of a rare disease, and symptoms $I_{a}, I_{b}$, and $I_{c}$ were imperfect predictors (associated with both a common disease and a rare disease). In the transfer stage that followed the training task, the use of base-rate information was investigated using tests with the imperfect predictors $\left(I_{a}, I_{b}, I_{c}\right)$, with two conflicting predictors (one of a common disease and one of a rare disease-i.e., $P C_{a} . P R_{a}, P C_{b} . P R_{b}$, or $P C_{c} . P R_{c}$ ), and with combined predictors $\left(I_{a} \cdot P C_{a} \cdot P R_{a}, I_{b} \cdot P C_{b} \cdot P R_{b}\right.$, or $I_{c} \cdot P C_{c}$. $P R_{c}$ ). Medin and Edelson reported the surprising result that participants preferred the less frequent category for the conflicting predictors (against the base rate), whereas they used base-rate information correctly for the imperfect predictors and for the combined predictors. The inverse base-rate effect has since been replicated in a number of studies, using different stimuli and procedures (e.g., Kalish, 2001; Kruschke, 1996; Winman, Wennerholm, Juslin, $\&$ Shanks, 2005).

Whereas the robustness of the inverse base-rate effect is not disputed, there is disagreement about the processes that underlie the effect. Two different kinds of explanations have been put forward. First, the effect has been attributed to context-dependent selective attention, operating within an otherwise normative learning and decision system. Probably the best example of such an account is
Kruschke's (1996) ADIT model. ADIT has been developed further since (e.g., Kruschke, 2001b), but the original model is appropriate for our present purposes. According to ADIT (which is an extension of Gluck \& Bower's, 1988, component-cue model), the inverse base-rate effect arises through selective attention during learning. Because the common disease occurs more frequently in the training phase, the symptoms associated with the common disease tend to be learned before those associated with the rare disease. Therefore, both the imperfect predictor $I$ and the perfect predictor $P C$ become associated with the common disease. When participants subsequently encounter the rare disease, attention moves away from the imperfect predictor $I$ (which is shared by both diseases) and becomes focused on the perfect predictor $P R$ (which is diagnostic for the rare disease). As a result, the representation of the common disease involves associative links from both $I$ and $P C$, whereas the rare disease will primarily be associated with the predictor $P R$. This asymmetry in the representation of the disease categories is sufficient to produce the inverse base-rate effect (Kruschke, 1996).

An alternative explanation of the inverse base-rate effect has been proposed by Juslin, Wennerholm, and Winman (2001). Juslin et al. identified a process (eliminative inference, which was formalized in the elimination model, or ELMO) that does not rely on asymmetric representations to produce the inverse base-rate effect. Instead, their account attributes the inverse base-rate effect to inferential processes that operate after learning, in the transfer stage. In ELMO, it is assumed that the participants learn inference rules that capture the structure of the task in the learning phase, by linking symptoms to diseases (e.g., I.PC $\rightarrow$ common). Because the common diseases occur more frequently, the rules that are associated with

K. Lamberts, k.lamberts@warwick.ac.uk 
Table 1

Abstract Representation of Training Task in Medin and Edelson (1988)

\begin{tabular}{ccc}
\hline Relative & & \\
Frequency & Symptoms & Disease \\
\hline 3 & $I_{a} \cdot P C_{a}$ & 1 \\
1 & $I_{a} \cdot P R_{a}$ & 2 \\
3 & $I_{b} \cdot P C_{b}$ & 3 \\
1 & $I_{b} \cdot P R_{b}$ & 4 \\
3 & $I_{c} \cdot P C_{c}$ & 5 \\
1 & $I_{c} \cdot P R_{c}$ & 6 \\
\hline
\end{tabular}

the common diseases become better established than the rules associated with the rare diseases. When a conflicting transfer stimulus $(P C . P R)$ is encountered, there is no perfect match with the conditions of any of the learned rules. The participants will notice in particular that there is no good match with the conditions of a well-established rule (i.e., a rule associated with a common disease). This triggers a process of eliminative inference, in which the common disease is rejected and the rare disease chosen. The eliminative inference process implements a metacognitive heuristic, which could be expressed informally as follows. "If these symptoms had been associated with the common disease, I would have noticed a good match with the conditions of a rule that I know very well. Because I did not notice a good match, it seems more likely that the symptoms were associated with a rule that I do not know so well, so I will choose the rare disease." A full description of ELMO can be found in Juslin et al. (2001).

Since the eliminative inference theory was proposed, there has been considerable debate about its merits and limitations, especially in comparison with the attention-shifting account (e.g., Kruschke, 2001a, 2003; Winman, Wennerholm, \& Juslin, 2003; Winman et al., 2005). Initially, the discussion centered primarily on the ability of both classes of models to explain response patterns in different versions of the base-rate task (e.g., Kruschke, 2003; Winman et al., 2003), without attempts to measure or manipulate the cognitive processes involved. In a recent article, Winman et al. (2005) did study the processes underlying the inverse baserate effect more directly. They demonstrated that younger children, who presumably do not have the metacognitive abilities required for eliminative inference, did not exhibit the inverse base-rate effect. Moreover, adults who were efficient learners (presumably due to their propensity for rule-based inference) or who preferred rule-based generalization in a separately administered learning task showed a stronger inverse base-rate effect than did other adults. On the basis of these results, Winman et al. (2005) concluded that rule-based processes play an essential role in producing the inverse base-rate effect. However, these conclusions were contradicted by the results of a series of experiments by Johansen, Fouquet, and Shanks (2007). Johansen et al. explored the conditions under which the inverse base-rate effect occurred by systematically manipulating the way in which information about the task was presented to the participants. If participants received only a summary description of the symptom-disease relations and the disease base rates (without going through an extensive training phase), the inverse base-rate effect did not occur. Systematic exploration of a continuum of presentation modes (from summary-based decision making to trial-by-trial learning) led Johansen et al. to conclude that asymmetric outcome representation and base-rate neglect are individually necessary and jointly sufficient to produce the inverse base-rate effect, without the need to assume an additional eliminative inference process.

In this article, we report a study whose aim was to shed further light on the cognitive processes that produce the inverse base-rate effect. As will be clear from the descriptions above, the attention-shifting model and the eliminative inference account differ not only in their representational assumptions, but also in their assumptions about the cognitive-processing load in the transfer stage of the task. In the attention-shifting model, transfer processing consists merely of an associative propagation of activation from symptom representations to disease representations. Such processing could be (largely) automatic and, thus, require minimal attentional resources (e.g., Logan, 1988; Moors \& De Houwer, 2006). Eliminative inference, on the other hand, almost certainly involves effortful, controlled, and capacity-demanding transfer processing. Juslin et al. (2001) state that "the implementation presented here (ELMO) is based on the idea that the participants perceive the task as one concerning the learning of a set of inference rules .... These rules are construed as conscious, high-level representations that are executed to the extent that they are active in working memory when a relevant probe is presented" (p. 851). It seems reasonable, therefore, to expect that eliminative inference is sensitive to interference from secondary tasks that compete for limited working memory capacity, whereas automatic associative processes should be immune (or, at least, less sensitive) to such interference. Secondary-load tasks have been used before to selectively suppress controlled inference processes, without affecting associative processes (e.g., De Houwer \& Beckers, 2003). If eliminative inference plays a role in the inverse base-rate effect, a secondary-load task should reduce or eliminate the effect. Alternatively, if the inverse base-rate effect is the result of associative processes operating on asymmetric cue-outcome representations, a secondary-load task should not reduce the effect.

Another manipulation that can be used to produce selective interference with high-level inference processes involves the time available for a decision in the transfer stage. If the participants have to respond very quickly, it is unlikely that they will have the time to carry out the different steps of an eliminative inference process (see Juslin et al., 2001, p. 853 , for a diagram of the processing stages involved). As a result, classification of stimuli that normally invoke eliminative interference (such as stimulus PC.PR) would be more affected by time pressure than would classification of stimuli that do not invoke eliminative inference (such as stimulus $I$ ). If eliminative inference is suppressed altogether, the inverse base-rate effect should even disappear, according to the eliminative inference account.

Time pressure would not have the same effect on associative processes. Although it is likely that fast responding would result in more errors overall (see Lamberts, 
1995, 1998), the output of associative processes would not change qualitatively, so that the inverse base-rate effect would only become weaker, without disappearing or reversing. Therefore, a reversal of the inverse base-rate effect under time pressure (i.e., toward base-rate-consistent responding for the conflicting stimuli) would be inconsistent with the associative account.

In the experiment reported in this article, we compared performance in a control condition (with the standard base-rate design) with performance under a secondary task load and with performance under two levels of time pressure. To avoid confounding effects of reading time and symptom presentation order (which would be particularly important in the conditions with time pressure), drawings of viruses (rather than verbal descriptions) were used as the stimulus materials.

\section{METHOD}

\section{Participants}

Thirty first-year psychology undergraduate students from the University of Warwick ( 24 women and 6 men, 18-39 years of age) participated as part of a course requirement.

\section{Materials and Stimuli}

The stimuli were presented on a 17 -in. CRT monitor with a resolution of $1,280 \times 1,024$ pixels and a refresh rate of $75 \mathrm{~Hz}$, viewed from a distance of $1 \mathrm{~m}$. Responses were made using the four right-hand buttons on a game pad, connected to the USB port of the control computer. The stimuli consisted of color images of virus-like objects. Every virus had a common outline and fill pattern, over which the other features were drawn. An example of four possible training stimuli is given in Figure 1 . The 18 possible transfer stimuli, given the feature instantiations in Figure 1, are shown in Figure 2. All the stimuli were presented in the center of the screen, against a uniform gray background.

\section{Design and Procedure}

The design was similar to that in Kruschke (1996, Experiment 1), except for the following differences: (1) A 7:1 common:rare ratio
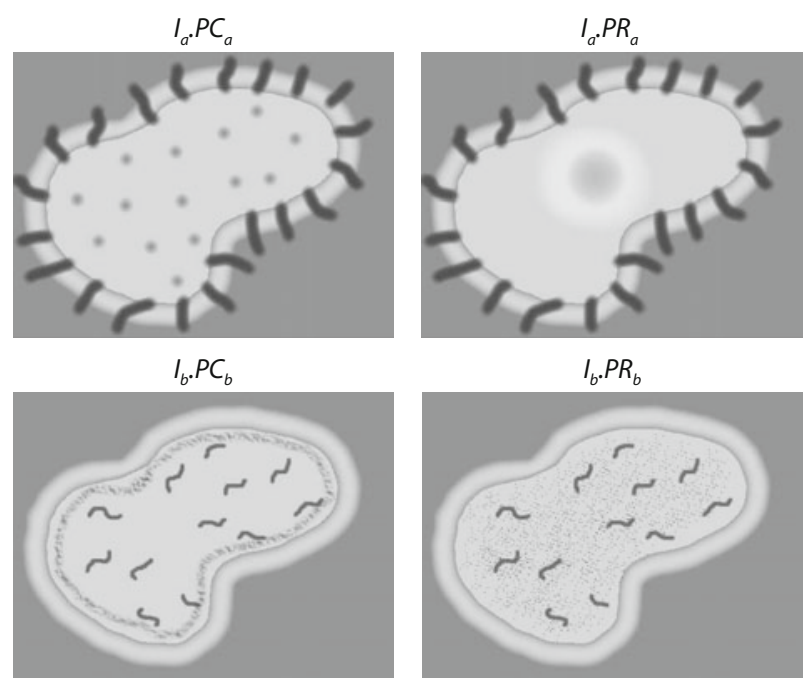

Figure 1. Example of four possible training stimuli. $I$, imperfect predictor; $P C$, perfect common predictor; $P R$, perfect rare predictor; $a$ and $b$ refer to two different stimulus instantiations that have the same abstract status. was used in training (following Shanks, 1992), instead of the 3:1 ratio in Kruschke (1996); (2) images were used as stimuli, instead of verbal descriptions; and (3) the participants were required to learn the training stimuli to a set performance criterion.

In the training phases of the experiment, four fictitious viruses were presented, with six variable features. Table 2 gives the abstract features of each of the four training viruses. Each of the four training viruses had an imperfect predictor $\left(I_{a}\right.$ or $\left.I_{b}\right)$ that was shared with one other training virus and a perfect predictor $\left(P C_{a}, P C_{b}, P R_{a}\right.$, or $\left.P R_{b}\right)$ that was not shared with any other training stimuli. In Table 2, two of the viruses are denoted common (their properties include either $P C_{a}$ or $P C_{b}$ ), and two are denoted rare (their properties include either $P R_{a}$ or $P R_{b}$ ). The common viruses were shown seven times more frequently than the rare viruses. The viruses subscripted $a$ had the same abstract structure as those subscripted $b$, differing only in the physical features assigned to the abstract features. There were two mapping schemes of six physical features to the six abstract features. Assignment of participants to the mapping schemes was randomized. The assignment of response buttons to training viruses was randomized for each participant. Eighteen novel combinations of the six features were presented in the transfer phase (these were the same 18 abstract-feature combinations as those used by Kruschke, 1996, Experiment 1).

Each participant took part in four conditions, called unspeeded, dual-task, 500-speeded, and 300-speeded, respectively. For each participant, the order of task completion was drawn (without replacement) from a randomized Latin square design. Each condition contained a training phase and a transfer phase. The training phase was identical in the four conditions. Learning in the training phase involved a sequential, supervised procedure. The participants were told that their task was to identify different viruses on the basis of their visual appearance. Presentation of training trials was randomized per block of 16 trials (containing one presentation of each rare virus and seven presentations of each common virus). In order to complete training and move on to a transfer phase, the participants needed to categorize a sequence of three blocks of viruses without errors. The first block of trials did not count toward this total. Each participant, therefore, completed a minimum of 48 trials in each training phase. Each training trial started with the presentation of a virus. The participants were then required to respond by pressing one of the four response buttons. Following the response, the virus disappeared and feedback was given. Feedback for correct responses consisted of the word "Correct!" printed in green Arial 24 font at the center of the screen and a high-frequency tone played through headphones. Feedback for incorrect responses consisted of the word "Wrong" printed in red 24-point Arial font at the center of the screen and a low-frequency tone. The visual feedback was presented for $1,000 \mathrm{msec}$. There was an intertrial interval of $1,750 \mathrm{msec}$.

In the transfer phase in each condition, the participants were told that they would see variants of the viruses they had just learned and that they should respond to these new viruses on the basis of their knowledge of the training viruses. In each transfer phase, the 18 different viruses were presented twice each, giving 36 transfer trials per condition. Presentation order was randomized per block of 36 trials. No feedback was given in the transfer phase.

The four conditions had different procedures in the transfer stages. In the transfer stage in the unspeeded condition, the participants could respond at their own pace. The transfer stage in the dual-task condition was identical to that in the unspeeded condition, except that the participants were required to count down from 1,000 in multiples of 3 throughout the transfer session. If participants miscalculated or paused for too long, the experimenter (who was present in the room) corrected or encouraged them to keep counting. In the transfer stage of the 500-speeded condition, the participants were required to respond immediately when they detected a response signal (a 100-msec tone), which was presented $500 \mathrm{msec}$ after the onset of the virus. If the participants responded more than $350 \mathrm{msec}$ after the tone onset, the words "Too Slow!" appeared in red Arial 24 font at the center of the screen. The 300 -speeded condition was identical to 




$I_{b}$

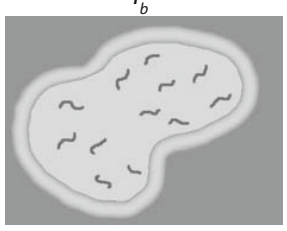

$P C_{a}$

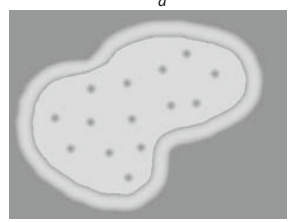

$P C_{b}$

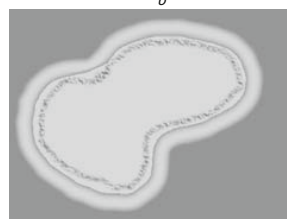

$P R_{a}$

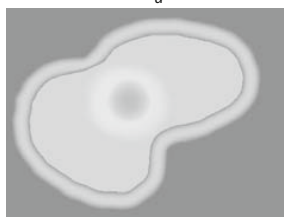

$P R_{b}$

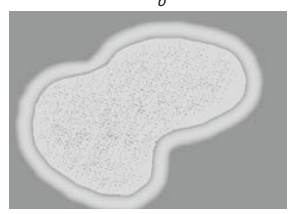



$P C_{b} \cdot P R_{b}$

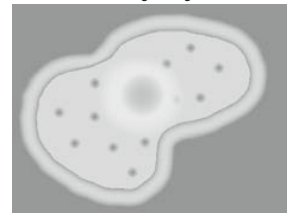

$I_{a} \cdot P C_{a} \cdot P R_{a}$

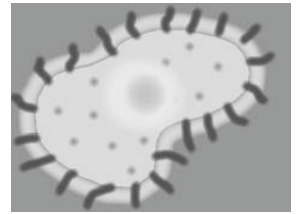

$I_{b} \cdot P C_{b} \cdot P R_{b}$



$I_{a} \cdot P C_{b}$

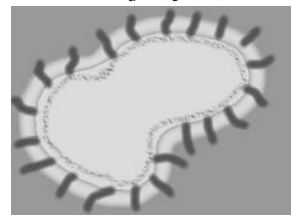

$I_{b} \cdot P C_{a}$

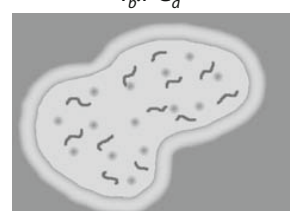

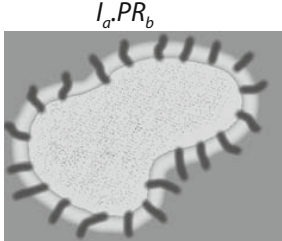

$I_{b} \cdot P R_{a}$

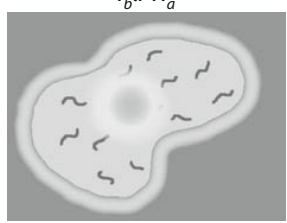

$P C_{a} \cdot P R_{b}$

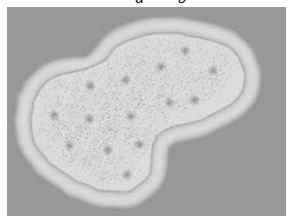

$P C_{b} \cdot P R_{a}$

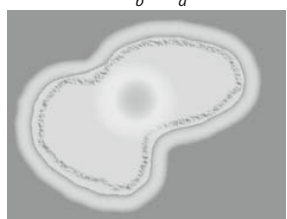

$I_{a} \cdot P C_{a} \cdot P R_{b}$



$I_{b} \cdot P C_{b} \cdot P R_{a}$



Figure 2. Example of 18 possible transfer stimuli. $I$, imperfect predictor; $P C$, perfect common predictor; $P R$, perfect rare predictor; $a$ and $b$ refer to two different stimulus instantiations that have the same abstract status.

the 500-speeded condition, except that the response signal occurred $300 \mathrm{msec}$ after onset of the virus.

\section{RESULTS}

The data from 4 participants who frequently failed to respond in time to the majority of the signals in the 500-speeded and 300-speeded conditions were removed from the analyses. For the remaining 26 participants, trials with responses that were made outside the 350-msec response windows were excluded from the analyses. Excluded trials constituted $2.5 \%$ and $10.8 \%$ of the total number of trials for the 500-speeded and 300-speeded conditions, respectively. We replicated the analyses of choice patterns without excluding these trials and found no meaningful differences from the analyses with excluded trials.

\section{Training}

A repeated measurements ANOVA showed a main effect of training phase on the number of blocks needed to reach criterion $\left[F(3,75)=3.19, M S_{\mathrm{e}}=10.24, p=.038\right]$. It took an average of 7.11 blocks $(S D=4.42)$ in the first training phase for the participants to reach criterion. The second phase required an average of 4.61 blocks $(S D=$ $1.77)$, the third phase took an average of 5.77 blocks $(S D=3.00)$, and in the fourth training phase the participants took an average of 5.92 blocks $(S D=3.69)$ to reach criterion. This improvement in learning rate suggests that 
Table 2

Feature Structure of the Training Stimuli

\begin{tabular}{lcccccc}
\hline & \multicolumn{6}{c}{ Feature } \\
\cline { 2 - 7 } \multicolumn{1}{c}{ Virus } & $I_{a}$ & $P C_{a}$ & $P R_{a}$ & $I_{b}$ & $P C_{b}$ & $P R_{b}$ \\
\hline Common $_{a}$ & 1 & 1 & 0 & 0 & 0 & 0 \\
Common $_{b}$ & 0 & 0 & 0 & 1 & 1 & 0 \\
$\operatorname{Rare}_{a}$ & 1 & 0 & 1 & 0 & 0 & 0 \\
Rare $_{b}$ & 0 & 0 & 0 & 1 & 0 & 1 \\
\hline
\end{tabular}

Note- $I$, imperfect predictor; $P C$, perfect common predictor; $P R$, perfect rare predictor; $a$ and $b$ refer to two different stimulus instantiations that have the same abstract status.

the participants were retaining information about the feature-category mapping across training phases.

\section{Transfer}

In the analyses, transfer data from instances of the same abstract structure were aggregated, to yield results for nine different stimulus types. For instance, the data from stimuli $I_{a}$ and $I_{b}$ (see Figure 2) were aggregated into abstract stimulus type $I$. As another example, the data from stimuli $I_{a} \cdot P C_{b}$ and $I_{b} \cdot P C_{a}$ were aggregated into abstract stimulus type $I . P C_{\mathrm{o}}$, in which the $o$ subscript (for "other") indicates a feature from the training stimuli with the alternative instantiation.

Before carrying out the main analyses of choice proportions for the different stimulus types, we analyzed response times (RTs) in the different conditions, to establish whether the load task and time pressure manipulations had been successful. A repeated measurements ANOVA (using Huynh-Feldt correction for violation of sphericity) with condition and stimulus (with nine levels; see Figure 3) as independent variables yielded significant main effects of condition $\left[F(3,75)=83.20, M S_{\mathrm{e}}=4,488,788\right.$, $p<.001]$ and stimulus $\left[F(8,200)=4.26, M S_{\mathrm{e}}=307,659\right.$, $p<.001]$ and a significant interaction between condition and stimulus $\left[F(24,600)=2.970, M S_{\mathrm{e}}=672,937, p=\right.$ $.004]$. Figure 3 shows an overview of the mean RTs in the four conditions. The main effect of condition indicates that the participants responded more quickly in the two speeded conditions than in the conditions without time pressure. Planned pairwise comparisons showed that all of the mean RTs in the four conditions differed from each other. RTs were longest in the dual-task condition $(M=$ 2,336 $\mathrm{msec}$ ), which indicates that the load task interfered with normal processing. RTs were significantly shorter in the two speeded conditions $(M=501 \mathrm{msec}$ in the 500 -speeded condition and $421 \mathrm{msec}$ in the 300-speeded condition) than in the unspeeded condition $(M=$ $1,297 \mathrm{msec}$ ), which indicates that the response signals did accelerate decision making. The main effect of stimulus and the interaction between stimulus and condition are potentially relevant. Unsurprisingly, the significant interaction reflects the larger RT differences between stimuli in the unspeeded and dual-task conditions than in the two speeded conditions. A comparison between the unspeeded and the dual-task conditions yielded main effects of task $\left[F(1,25)=31.39, M S_{\mathrm{e}}=4,022,860, p<.001\right]$ and stimulus $\left[F(8,200)=4.34, M S_{\mathrm{e}}=622,861, p<.001\right]$, but no interaction between task and stimulus $[F(8,200)=2.06$, $p=.073]$. The question remains as to what the RT differences between the stimuli in the unspeeded conditions can tell us about processing in the task. We will return to this issue in the Discussion section.

The choice data from the four conditions are shown in the Appendix (Tables A1-A4). Most of the discussion

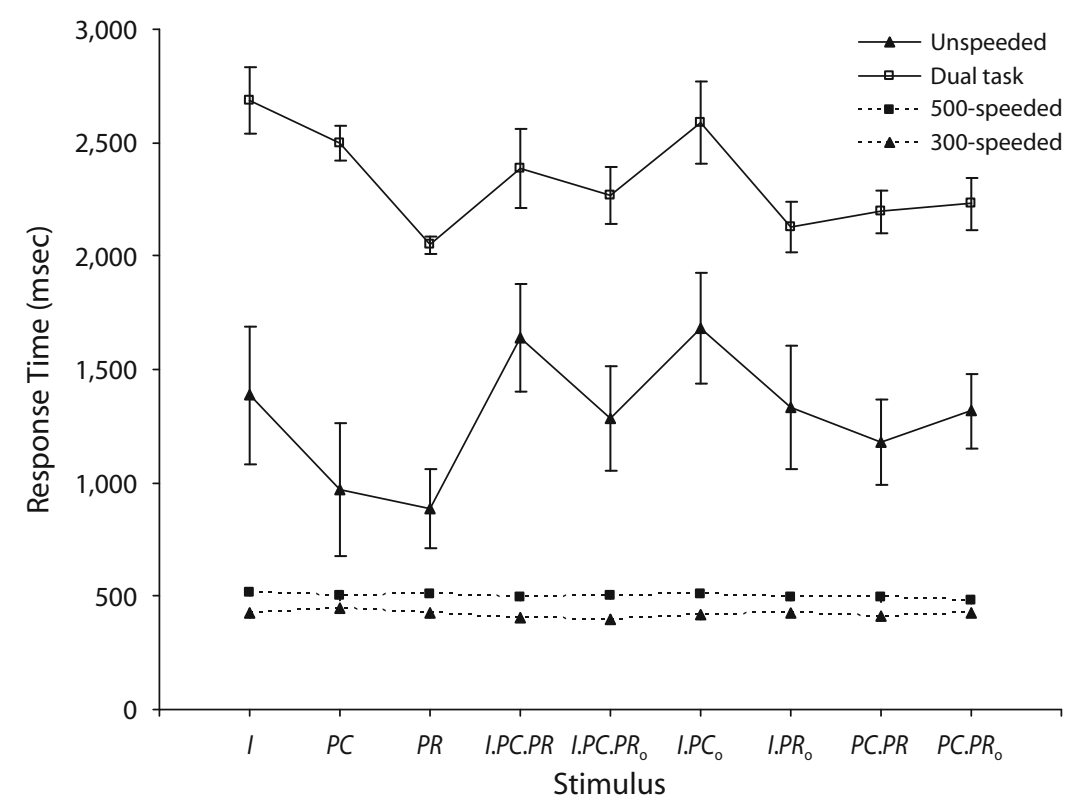

Figure 3. Mean response times (in milliseconds) in the four conditions. The error bars show standard errors. $I$, imperfect predictor; $P C$, perfect common predictor; $P R$, perfect rare predictor. The $o$ subscript (for "other") indicates a feature from the training stimuli with the alternative instantiation. 
here will focus on the data for three stimuli. A diagnostic result for the inverse base-rate effect concerns the difference between the responses to stimulus types $I$ and PC.PR (and the equivalent difference between the stimulus types $I$ and $P C . P R_{\mathrm{o}}$ ). A typical finding is that stimulus $I$ produces a majority of common category responses (consistent with the base rate), whereas $P C . P R$ and $P C . P R_{\mathrm{o}}$ tend to produce more rare category responses (against the base rate, thus producing the core effect; e.g., Kruschke, 1996; Medin \& Edelson, 1988). Because stimulus $I$ is the simplest stimulus, and because it has been paired with the common and rare categories with frequencies that correspond to the base rates of the categories, it provides an excellent reference point for evaluating response patterns to the other stimuli. The comparisons between the stimuli were based on the response frequencies across the four possible response categories. The frequency distributions for different stimuli were compared using a Pearson chisquare test of association. Where appropriate, the tests were based on realigned response categories, to ensure that only formally equivalent proportions were compared. For instance, the four response categories for $P C . P R$ would be common, rare, common $_{\mathrm{o}}$, and rare $_{\mathrm{o}}$; the corresponding realigned categories for $P C . P R_{\mathrm{o}}$ would be common, rare ${ }_{\mathrm{o}}$, common $_{\mathrm{o}}$, and rare, respectively.

In the unspeeded condition, the choice pattern for stimulus $I$ differed significantly from the pattern for PC.PR $\left[\chi^{2}(3)=33.29, p<.001\right]$ and from the realigned pattern for $P C . P R_{\mathrm{o}}\left[\chi^{2}(3)=30.42, p<.001\right]$. The same differences were significant in the dual-task condition $\left[\chi^{2}(3)=\right.$ $24.00, p<.001$, and $\chi^{2}(3)=21.39, p<.001$, respectively]. In the 500-speeded condition, there were also significant differences between $I$ and $P C . P R\left[\chi^{2}(3)=16.26\right.$, $p=.001]$ and between $I$ and realigned $P C . P R_{\mathrm{o}}\left[\chi^{2}(3)=\right.$ $11.40, p<.01]$. In the 300 -speeded condition, the same differences emerged $\left[\chi^{2}(3)=11.64, p<.01\right.$, and $\chi^{2}(3)=$ $22.43, p<.001$, respectively].

Whereas the results of the chi-square tests confirmed that choice patterns differed reliably between stimulus $I$ and stimulus PC.PR (or PC.PR ) in each condition, it was still necessary to confirm that these differences represented an inverse base-rate effect. Figure 4 shows an overview of the critical choice proportions. In each of the four conditions, the participants preferred the common response for the $I$ stimuli and the rare (or rare $_{\mathrm{o}}$ ) response for the PC.PR and PC.PR $R_{\mathrm{o}}$ stimuli, confirming the standard
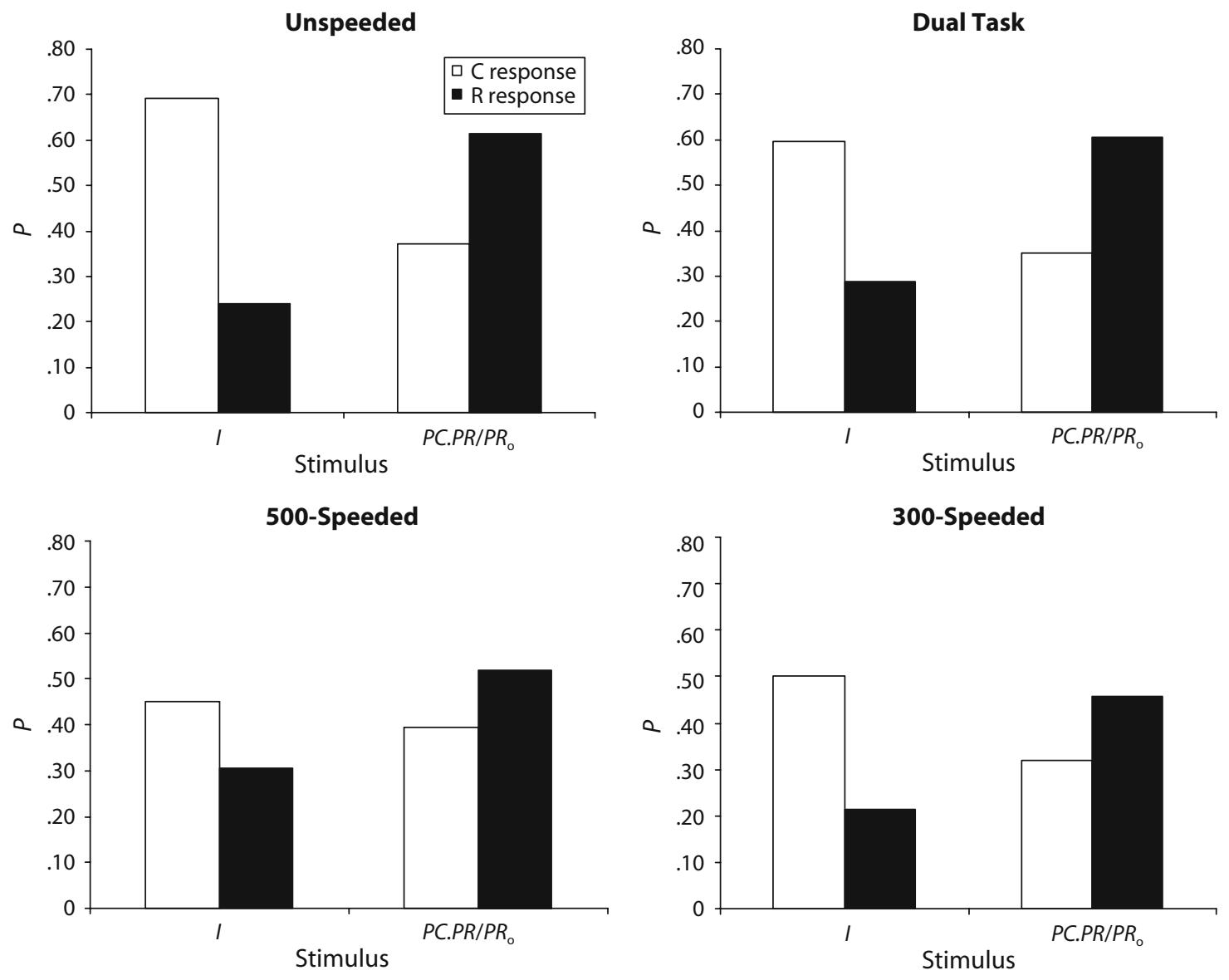

Figure 4. Proportions of common $(C)$ and rare (R) responses to stimulus $I$ and stimuli $P C . P R$ and $P C$. $P R_{0}$. The average of the proportions for $P C . P R$ and $P C . P R_{0}$ is shown. For $P C . P R_{0}$, the proportion of $R_{0}$ responses was used in the calculation of the average rare proportion. 
inverse base-rate result (e.g., Juslin et al., 2001; Kalish, 2001; Kruschke, 1996; Winman et al., 2005).

The inverse base-rate effect persisted under dual-task and time pressure manipulations that were sufficiently strong to affect the participants' task performance in other ways. We have already demonstrated that the load task induced a significant increase in RTs. In the two speeded conditions, the time pressure affected response accuracy for most of the stimuli. For instance, stimulus $P C$ (which had been associated only with the common category) produced $90 \%$ common responses in the unspeeded condition, but only $73 \%$ in the 500 -speeded condition and $63 \%$ in the 300-speeded condition. Similarly, performance on $P R$ (which had been associated only with the rare category) dropped from $96 \%$ correct in the unspeeded condition to $76 \%$ in the 500 -speeded condition and $63 \%$ in the 300 -speeded condition. Even under time pressure that was sufficient to increase error rates for most stimuli, the participants still preferred the rare category for the conflicting transfer stimuli.

\section{DISCUSSION}

The choice data show a clear pattern: The inverse base-rate effect occurred in the presence of a demanding secondary task, which has been shown to interfere with controlled high-level processes (e.g., Dean, Dewhurst, Morris, \& Whittaker, 2005; Engelkamp \& Zimmer, 1996), and under severe levels of time pressure. The results from the two speeded conditions have direct implications for accounts of the inverse base-rate effect in terms of rulebased inference processes, such as those provided in Juslin et al.'s (2001) ELMO. Under levels of time pressure that were sufficiently severe to interrupt normal processing (as evident from higher error rates across stimuli), the inverse base-rate effect persisted. It is difficult to reconcile this result with the operation of an elaborate, controlled inference process, as is proposed in ELMO. Alternatively, an associative, attention-shifting account (such as that provided by Kruschke, 1996) would have no difficulties explaining the data from the speeded conditions.

The dual-task condition showed that the choice patterns under secondary task load were almost indistinguishable from those without a secondary load, which could be taken as further evidence against ELMO. However, the secondary task increased mean RTs by more than $1 \mathrm{sec}$, as compared with the unspeeded condition. The locus of the interference effect is not clear, but it could be argued that the longer RTs in the dual-task condition provide evidence for the operation of a capacity-demanding process in the main task (not necessarily the process postulated in ELMO, but a process that nonetheless engages executive function). However, the secondary task had a similar effect on RTs to all the stimuli (there was no significant interaction between stimulus and condition when only the unspeeded and dual-task conditions were included in the analysis). This pattern of RTs suggests that the interference in the dual-task condition affected a stage of processing common to all the stimuli. If the secondary task interfered with the eliminative inference processes postu- lated in ELMO, the RTs to stimuli for which ELMO predicts high rates of eliminative inference (such as PC.PR) should be affected more than RTs to stimuli with low rates of eliminative inference (such as stimulus $I$, which would be processed primarily via the much simpler process of direct induction). Therefore, the RT data do not provide any evidence that eliminative inference occurred.

Regardless of the secondary-task interference, there were significant RT differences between the stimuli. In the unspeeded condition, there was a difference of nearly 800 msec between the shortest RT (to stimulus $P R$ ) and the longest RT (to stimulus $I . P C_{\mathrm{o}}$ ). It is difficult to derive predictions of RT differences between stimuli from ELMO without making speculative assumptions about the duration of the different processes in the model. Likewise, Kruschke's (1996) ADIT model was not designed to predict RTs. However, it is fairly straightforward to derive RT predictions from ADIT. After simulated training, ADIT will have produced association weights between each symptom and each category. For instance, in Kruschke's (1996) Experiment 1, the best-fitting association weight from symptom $P C$ to Category $\mathrm{C}$ was .717 , and the association weight from $P C$ to $\mathrm{R}$ was -.224 . By adding the association weights from the symptoms in a given stimulus to the relevant response categories, the total evidence for each response category can be computed. It is well known that RTs in categorization tasks depend on the relative evidence for the categories that is provided by the stimulus. If there is strong evidence for one category and little evidence for another, RTs will tend to be short (e.g., Lamberts, 2000; Nosofsky \& Palmeri, 1997). By computing the difference between the evidence values for the two most relevant response categories for each stimulus, ADIT can make specific RT predictions, and the RT data from our experiment could, therefore, be informative about the validity of the attention-shifting account.

To test whether the RT data from the unspeeded condition were consistent with the predictions derived from ADIT, we constructed a simple linear model of RTs that used the relative evidence values derived from ADIT for Experiment 1 in Kruschke (1996, Table 11, p. 17). The relative evidence values were linearly transformed to produce RT predictions. The model thus had only two free parameters: a slope and an intercept. This model was fitted to the RTs from the unspeeded condition. A leastsquares criterion was used to find the best-fitting values of the two model parameters. The estimated intercept was $1,566 \mathrm{msec}$, and the estimated slope was -624 . Figure 5 shows an overview of observed and predicted RTs. The model predicted the main trends in the RT data well $(r=$ .85 ), considering that the estimated parameters only had a scaling function and did not alter the correlation between the relative evidence values and the observed RTs. Therefore, the RT data from the unspeeded condition provided further support for the attention-shifting account.

The results from our experiment appear to contradict the conclusions from Winman et al.'s (2005) study, in which they demonstrated that children do not show a significant inverse base-rate effect and in which adults showed considerable individual differences in processing strategy. 




Figure 5. Observed and predicted mean response times (in milliseconds) for each stimulus in the transfer task of the unspeeded condition. Obs, observed; Pred, predicted.

However, it is possible that the children in Winman et al.'s (2005) study did not show the effect because they relied on associative processes that were somehow different from those employed by most adults. Perhaps the absence of an inverse base-rate effect in children was the result of an inability to shift attention when necessary. The adult data from Winman et al. (2005) indicate that people can use different strategies to complete the task, which raises the questions of why and when different strategies might be preferred. It is possible, for instance, that the nonverbal materials in our experiment discouraged the participants from exploring a rule-based approach. Still, the results from our experiment (particularly from the two speeded conditions) indicate that elaborate, rule-based processing is not essential for generating the inverse base-rate effect.

The main conclusion from the experiment reported in this article is that we found no evidence for the operation of controlled rule-based processes in the inverse base-rate task. The inverse base-rate effect persisted in conditions in which the time pressure was sufficiently severe to produce a general decline in classification performance and in the condition with a demanding secondary task. We conclude that it is not necessary to postulate that high-level metacognitive processes are responsible for the inverse base-rate effect.

\section{AUTHOR NOTE}

This research was supported by Biotechnology and Biological Sciences Research Council Grant BBS/B/08914 (with K.L. as PI). We thank three anonymous reviewers for their helpful comments on a previous version of the manuscript. Correspondence concerning this article should be addressed to K. Lamberts, Department of Psychology, University of Warwick, Coventry CV4 7AL, England (e-mail: k.lamberts@warwick.ac.uk).

\section{REFERENCES}

Dean, G. M., Dewhurst, S. A., Morris, P. E., \& Whittaker, A. (2005). Selective interference with the use of visual images in the symbolic distance paradigm. Journal of Experimental Psychology: Learning, Memory, \& Cognition, 31, 1043-1068.

De Houwer, J., \& BecKers, T. (2003). Secondary task difficulty modulates forward blocking in human contingency learning. Quarterly Journal of Experimental Psychology, 56B, 345-357.

ENGELKAMP, J., \& ZiMmeR, H. (1996). Organisation and recall in verbal tasks and in subject-performed tasks. European Journal of Cognitive Psychology, 8, 257-273.

Gluck, M. A., \& Bower, G. H. (1988). From conditioning to category learning: An adaptive network model. Journal of Experimental Psychology: General, 117, 227-247.

Johansen, M. K., FouQuet, N., \& Shanks, D. R. (2007). Paradoxical effects of base rates and representation in category learning. Memory \& Cognition, 35, 1365-1379.

Juslin, P., Wennerholm, P., \& Winman, A. (2001). High-level reasoning and base-rate use: Do we need cue-competition to explain the inverse base-rate effect? Journal of Experimental Psychology: Learning, Memory, \& Cognition, 27, 849-871.

KaLISH, M. L. (2001). An inverse base rate effect with continuously valued stimuli. Memory \& Cognition, 29, 587-597.

KRUSCHKE, J. K. (1996). Base rates in category learning. Journal of Experimental Psychology: Learning, Memory, \& Cognition, 1, 3-26.

KrUSChKE, J. K. (2001a). The inverse base-rate effect is not explained by eliminative inference. Journal of Experimental Psychology: Learning, Memory, \& Cognition, 27, 1385-1400.

KRUSCHKE, J. K. (2001b). Toward a unified model of attention in associative learning. Journal of Mathematical Psychology, 45, 812-863.

KruschKe, J. K. (2003). Attentional theory is a viable explanation of the inverse base-rate effect: A reply to Winman, Wennerholm, and Juslin (2003). Journal of Experimental Psychology: Learning, Memory, \& Cognition, 29, 1396-1400.

LamberTs, K. (1995). Categorization under time pressure. Journal of Experimental Psychology: General, 124, 161-180.

LAMBERTS, K. (1998). The time course of categorization. Journal of Experimental Psychology: Learning, Memory, \& Cognition, 24, 695-711.

LAMBERTs, K. (2000). Information-accumulation theory of speeded categorization. Psychological Review, 107, 227-260.

Logan, G. D. (1988). Toward an instance theory of automatization. Psychological Review, 95, 492-527.

Medin, D. L., \& Edelson, S. M. (1988). Problem structure and the use of base-rate information from experience. Journal of Experimental Psychology: General, 117, 68-85. 
Moors, A., \& De Houwer, J. (2006). Automaticity: A theoretical and conceptual analysis. Psychological Bulletin, 132, 297-326.

Nosofsky, R. M., \& PALMeri, T. J. (1997). An exemplar-based random walk model of speeded classification. Psychological Review, 104, 266-300.

SHANKs, D. R. (1992). Connectionist accounts of the inverse base-rate effect in categorization. Connection Science, 4, 3-18.
Winman, A., Wennerholm, P., \& Juslin, P. (2003). Can attentional theory explain the inverse base-rate effect? Comments on Kruschke (2001). Journal of Experimental Psychology: Learning, Memory, \& Cognition, 29, 1390-1395.

Winman, A., Wennerholm, P., Juslin, P., \& Shanks, D. R. (2005). Evidence for rule-based processes in the inverse base-rate effect. Quarterly Journal of Experimental Psychology, 58A, 789-815.

\section{APPENDIX}

Table A1

Response Proportions for Transfer Stimuli in Unspeeded Condition

\begin{tabular}{lcccc}
\hline \multirow{2}{*}{\multicolumn{1}{c}{ Virus }} & \multicolumn{4}{c}{ Category } \\
\cline { 2 - 5 } I & Common & Rare & Common $_{\mathrm{o}}$ & Rare $_{\mathrm{o}}$ \\
$P C$ & .69 & .24 & .03 & .04 \\
$P R$ & .89 & .03 & .08 & .00 \\
$P C . P R$ & .04 & .96 & .00 & .00 \\
$I . P C . P R$ & .37 & .63 & .01 & .00 \\
$P C . P R_{\mathrm{o}}$ & .57 & .42 & .00 & .01 \\
$I . P C . P R_{\mathrm{o}}$ & .38 & .00 & .02 & .61 \\
$I . P C_{\mathrm{o}}$ & .62 & .00 & .00 & .38 \\
$I . P R_{\mathrm{o}}$ & .38 & .04 & .58 & .01 \\
\hline & .25 & .02 & .00 & .73 \\
\hline
\end{tabular}

Note- $I$, imperfect predictor; $P C$, perfect common predictor; $P R$, perfect rare predictor. The $o$ subscript (for “other") indicates a feature from the training stimuli with the alternative instantiation.
Table A2

Response Proportions for Transfer Stimuli in Dual-Task Condition

\begin{tabular}{lcccc}
\hline \multirow{2}{*}{ Virus } & \multicolumn{4}{c}{ Category } \\
\cline { 2 - 5 }$I$ & Common & Rare & Common $_{\mathrm{o}}$ & Rare $_{\mathrm{o}}$ \\
$P C$ & .60 & .29 & .08 & .04 \\
$P R$ & .80 & .05 & .09 & .07 \\
$P C . P R$ & .03 & .93 & .01 & .03 \\
$I . P C . P R$ & .33 & .63 & .03 & .02 \\
$P C . P R_{\mathrm{o}}$ & .41 & .54 & .01 & .04 \\
$I . P C . P R_{\mathrm{o}}$ & .38 & .03 & .01 & .59 \\
$I . P C_{\mathrm{o}}$ & .48 & .02 & .01 & .49 \\
$I . P R_{\mathrm{o}}$ & .38 & .08 & .50 & .05 \\
\hline
\end{tabular}

Note $-I$, imperfect predictor; $P C$, perfect common predictor; $P R$, perfect rare predictor. The $o$ subscript (for "other") indicates a feature from the training stimuli with the alternative instantiation.
Table A3

Response Proportions for Transfer Stimuli in 500-Speeded Condition

\begin{tabular}{lcccc}
\hline \multirow{2}{*}{ Virus } & \multicolumn{4}{c}{ Category } \\
\cline { 2 - 5 }$I$ & Common & Rare & Common $_{\mathrm{o}}$ & Rare $_{\mathrm{o}}$ \\
$P C$ & .45 & .31 & .15 & .09 \\
$P R$ & .73 & .09 & .08 & .10 \\
$P C . P R$ & .11 & .76 & .09 & .04 \\
$I . P C . P R$ & .40 & .53 & .04 & .03 \\
$P C . P R_{\mathrm{o}}$ & .39 & .47 & .10 & .05 \\
$I . P C . P R_{\mathrm{o}}$ & .38 & .05 & .06 & .51 \\
$I . P C_{\mathrm{o}}$ & .48 & .04 & .08 & .40 \\
$I . P R_{\mathrm{o}}$ & .29 & .10 & .52 & .09 \\
\hline
\end{tabular}

Note- $I$, imperfect predictor; $P C$, perfect common predictor; $P R$, perfect rare predictor. The $o$ subscript (for "other") indicates a feature from the training stimuli with the alternative instantiation.
Table A4

Response Proportions for Transfer Stimuli in 300-Speeded Condition

\begin{tabular}{lcccc}
\hline \multirow{4}{*}{ Virus } & \multicolumn{4}{c}{ Category } \\
\cline { 2 - 5 } & Common & Rare & Common $_{\mathrm{o}}$ & Rare $_{\mathrm{o}}$ \\
\hline$I$ & .50 & .21 & .20 & .09 \\
$P C$ & .63 & .11 & .16 & .11 \\
$P R$ & .20 & .63 & .06 & .10 \\
$P C . P R$ & .30 & .43 & .18 & .08 \\
$I . P C . P R$ & .41 & .35 & .15 & .09 \\
$P C . P R_{\mathrm{o}}$ & .33 & .14 & .05 & .48 \\
$I . P C . P R_{\mathrm{o}}$ & .39 & .11 & .14 & .36 \\
$I . P C_{\mathrm{o}}$ & .41 & .12 & .37 & .10 \\
$I . P R_{\mathrm{o}}$ & .26 & .05 & .22 & .47 \\
\hline
\end{tabular}

Note $-I$, imperfect predictor; $P C$, perfect common predictor; $P R$, perfect rare predictor. The $o$ subscript (for "other") indicates a feature from the training stimuli with the alternative instantiation. 